EPJ Web of Conferences 19, 10005 (2012)

DOI: $10.1051 /$ epjconf/20121910005

(C) Owned by the authors, published by EDP Sciences, 2012

\title{
Preparing the Besançon Galaxy model for the comparison with Gaia data
}

\author{
M. Czekaj ${ }^{1, a}$, A.C. Robin ${ }^{2}$, X. Luri ${ }^{1}$ and F. Figueras ${ }^{1}$ \\ ${ }^{1}$ Departament d'Astronomia i Meteorologia, ICC-UB and IEEC, Barcelona, Spain \\ 2 Institut Utinam, CNRS UMR6213, Université de Franche-Comté, Observatoire de Besançon, \\ Besançon, France
}

\begin{abstract}
From the Gaia mission, kinematic and star count data, together with the physical parameters of the stars - ages and metallicities-, will allow to characterise our Galaxy populations and, from that, the overall Galactic gravitational potential. One of the promising procedures to reach such goal will be to optimise the present Population Synthesis models by fitting, through robust statistical techniques, the large and small scale structure and kinematics parameters that best will reproduce Gaia data. As a first step we present here the comparison between the data from the Tycho- 2 catalogue and the Besançon Galaxy Model simulations, as well as our present our work on the model optimization.
\end{abstract}

\section{INTRODUCTION}

The Besançon Galaxy Model is a stellar population synthesis model, built over the last two decades in Besançon, [7] and [8]. Until now the stars production process was based on the drawing from so called Hess diagrams. Each Galaxy population had one such a diagram, which was calculated once given a particular IMF, SFR and evolutionary tracks and since then remained fixed throughout all simulations. In this $\mathrm{PhD}$ project we are constructing a new version of the Besançon Galaxy Model able to handle variations of the star formation rate in different populations, initial mass function and evolutionary tracks. This requires the Galactic gravitational potential recalculation in order to accomplish the dynamical self-consistency. The process of model's optimization is still ongoing. Here we present the first comparison of the new model with Tycho-2 data. In our simulations we have applied various scenarios for the IMF and SFR, different evolutionary tracks sets, different atmosphere models and different values for dynamical mass and the age- $\sigma_{W}$ relation. In the future of this research project we plan to improve the kinematical description of the model's thin disc.

\section{BESANÇON GALAXY MODEL VS. TYCHO-2}

The Tycho-2 catalogue [5] was chosen to be our primary data set used for intensive comparisons with simulations. The complete sample of stars until magnitude $V_{T}=11.0$ was extracted for our analysis also checking if the proper motions were available. The number of objects selected for our analysis was 860743. The Tycho-2 photometry was converted to the Johnson - Cousin photometric system.

As the first step we have developed a processing pipeline, which allowed us to perform a whole sky comparison between the model and the data. The sky was divided into 472 fields and for each region we have compared the star counts, B-V colour and proper motion distributions applying different extinction

ae-mail: mczekaj@am.ub.es

This is an Open Access article distributed under the terms of the Creative Commons Attribution-Noncommercial License 3.0, which permits unrestricted use, distribution, and reproduction in any noncommercial medium, provided the original work is properly cited. 


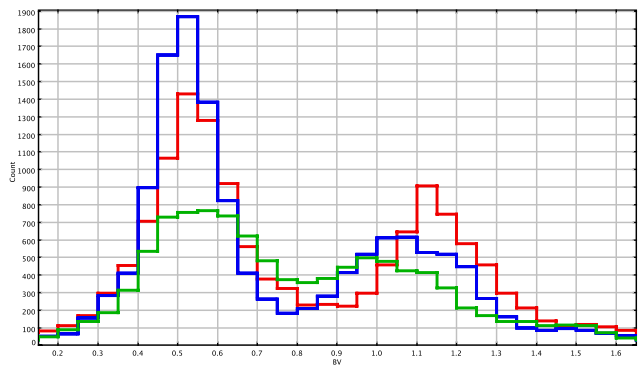

a) B-V distribution: change in the evolutionary tracks

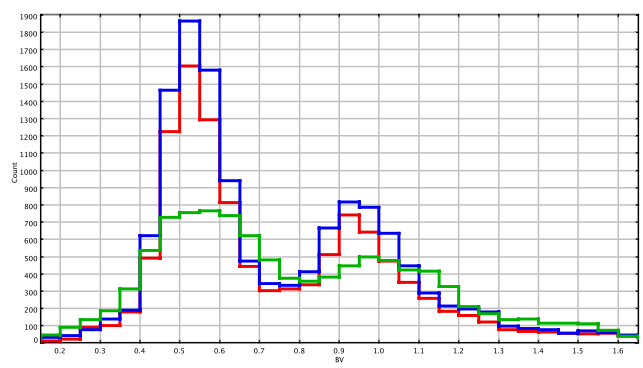

b) B-V distribution: change in the IMF

Figure 1. The comparison of model's and Tycho-2 B-V distributions towards the Galactic North Pole; a) when two different packages of evolutionary tracks were applied in simulations, in red the BGM with the old tracks, in blue the BGM with newer sets and in green the Tycho-2 data, check the text below for the references; $b$ ) when two different IMF scenarios were imposed in the model, in red the IMF specified in [7], in blue the IMF from [6] and in green Tycho-2 data.

models. The results from all single regions served us to produce the whole sky plots and derive the global conclusion, that BGM and Tycho show significant differences. The fundamental discrepancies were concerning the B-V distribution, they were detected at the level of single field analysis and clearly defined in the cumulative all sky B-V distribution. We chose those discrepancies to be the departure point of our investigation and during our research we have checked how we can improve the B-V distributions, when different scenarios for various model's inputs are imposed.

After rearranging the model's code the first change we have made was the replacement of the evolutionary tracks package used for simulations. From the old one described in [8] we moved to the newer version, which we built up from [2] for the low masses and a compilation of [1], [3] and [4] for the high masses. In the Fig. 1 a) we present you the comparison of B-V distributions towards the Galactic North Pole, when those two evolutionary tracks sets were applied in simulations. In red the BGM with the old tracks, in blue the BGM with newer sets and in green the Tycho- 2 data. One can notice an improvement in the red peak of the distribution, which refers to giants. We conclude that it was obtained due to both, the change in the model's code arrangement and in the evolutionary tracks. The same methodology of changing the value of model's input parameter, repeating the simulations and comparing them with the old ones and the Tycho-2 data, was used in case of atmosphere models, the SFR, dynamical mass, age- $\sigma_{W}$ relation and the IMF. As an another example, in the Fig. $1 \mathrm{~b}$ ) we show how the North Galactic Pole B-V distribution changed, when the IMF was modified. In red the IMF specified in [7], in blue the IMF from [6] and in green Tycho-2 data.

We find our new model a powerful tool. It allows us to study the star formation processes, which ocurred throughout the age of the thin disc of our Galaxy. Our project continues and it is oriented to testing several scenarios for model's inputs, checking the correlations between model's ingredients and finding for them values, which reproduces data best.

\section{References}

[1] Bressan A., Fagotto F., Bertelli G., Chiosi C., A\&A, 100, 1993 647-664

[2] Chabrier G., Baraffe I., A\&A, 327, 1997 1039-1053

[3] Fagotto F., Bressan A., Bertelli G., Chiosi C., A\&A, 104, 1994a 365-376

[4] Fagotto F., Bressan A., Bertelli G., Chiosi C., A\&A, 105, 1994b 29-38 
Assembling the Puzzle of the Milky Way

[5] Høg, E. and Fabricius, C. and Makarov, V. V. and Urban, S. and Corbin, T. and Wycoff, G. and Bastian, U. and Schwekendiek, P. and Wicenec, A., A\&A, 355, 27-30

[6] Just, A., Jahreiß, H., A\&A, 402, 2010 461-478

[7] Robin, A., C. and Creze, M., A\&A, 157, 1986 71-90

[8] Robin, A., C., Reylé, C., Derrière, S. and Picaud, S., A\&A, 409, 2003 523-540 